\title{
Desastres antrópicos en las Tradiciones Peruanas
}

\author{
Lorenzo Huertas Vallejos \\ Universidad Ricardo Palma \\ lhuertas@urp.edu.pe \\ Lima-Perú
}

\section{Resumen}

Los desastres que alteran el desarrollo social son de dos tipos: naturales y sociales; Ricardo Palma registró en sus Tradiciones Peruanas y también en sus epístolas ambos tipos de fenómenos. Este artículo examina en la obra palmista los desastres ocasionados por el hombre, o antrópicos. Para esta indagación, hemos seleccionado la tradición "Los Caballeros de la Capa. Crónica de una guerra civil" que trata de un hecho que marcó los destinos del Perú: la guerra entre Francisco Pizarro y Diego de Almagro.

Palabras clave: desarrollo social, tradiciones, Ricardo Palma, Francisco Pizarro, Diego de Almagro.

\section{Abstract:}

The disasters that alter the social development are of two types: natural and social. Ricardo Palma registered both types phenomenons in his Peruvian Traditions and also in his epistles. This article examines disasters caused by the man or anthropics in the work of Palma. For this inquiry, we have selected the tradition "Los Caballeros de la Capa. Crónica de una guerra civil" which deals with an event that marked the destiny of Peru: the war between Francisco Pizarro and Diego de Almagro.

Keywords: Social development, traditions, Ricardo Palma, Francisco Pizarro, Diego de Almagro. 
Lorenzo Huertas Vallejos (Perú): Doctorado en la Universidad Mayor de San Marcos. Miembro de número de la Academia de Historia del Perú, profesor emérito de la Universidad Nacional San Cristóbal de Humanaga, miembro de número de la sociedad Bolivariana del Perú, miembro de número del Instituto Ricardo Palma. Director del Archivo de la URP. 


\section{Introducción}

En la historia del Perú se registran desastres severos que han afectado el normal desarrollo de la sociedad; estos - como ya se dijo- son de dos tipos: 1) desastres naturales y 2) desastres antrópicos o sociales. Ricardo Palma en sus tradiciones menciona estos dos tipos de desastres. En artículos publicados en la revista Aula Palma hemos tratado sobre desastres naturales encontrados en las referidas tradiciones. Estos registros que hizo Palma abarcan una larga cronología: comienzan en tiempos de los incas que gobernaron el Tahuantinsuyo y se prolongan hasta las segunda mitad del siglo XIX. Palma entendía plenamente que la recurrencia que estos fenómenos naturales (diluvios en el norte del Perú, terremotos o erupciones volcánicas en el sur, aparición de pandemias por distintas regiones peruanas amen de fenómenos celestiales inesperados) cincelaban el carácter de los pueblos y que, por lo tanto, había que registrarlos. También hemos dicho que gracias a este registro de desastres hecho por Palma hemos podido confirmar la existencia de los llamados lapsos críticos o tiempos de convergencia de desastres de diversa naturaleza, casi simultáneos o muy próximos unos de otros; nos referimos a fenómenos del Niño, sequías, terremotos erupciones volcánicas, epidemias, plagas, etc. Con estas ricas referencias pudimos publicar el libro Las Injurias del tiempo en el año 2009. Ahora nos proponemos hacer una selección de los desastres sociales o antrópicos que han sido tratados por la historia, pero para encontrar detalles que solo Palma pudo hallar, hemos creído conveniente analizarlos y exponerlos conforme lo hicimos con los desastres naturales.

\section{Registros de desastres sociales en la obra de Palma}

En un primer sondeo de las tradiciones y del epistolario de Palma encontramos la tradición "Los caballeros de la capa. Crónica de 
una guerra civil", que aparece en el primer tomo, volumen 1 de la primera y segunda parte de las Tradiciones peruanas, en la edición publicada recientemente por la Universidad Ricardo Palma (2014). En dicha tradición, nuestro escritor trata un hecho nodal en la historia del Perú: el asesinato de Francisco Pizarro, evento que a la vez originó otros sucesos tan singulares como el hecho matriz. Antes de pasar al análisis de la referida tradición conviene tener en cuenta lo siguiente:

Cuando tratamos de los desastres naturales notamos que la presencia de estos afectaban y afectan la geopolítica, originan retracción económica; además producen cambios en la ideología; por lo general para el imaginario popular aparecen las patologías del fin del mundo y hay estrés genérico, de igual manera hay cambios en el comportamiento de la gente, algunos se inclinan por la autogénesis mientras otros se aferran a la etnogénesis. Entonces, la pregunta que resulta es ¿cómo se ve afectada la sociedad ante un desastre antrópico? y icómo Palma los registra?

Además, debemos tener en cuenta que el Perú es uno de los países de América que registra a lo largo de su historia el mayor número de conflictos sociales; y, sin duda, tal recurrencia es una de las causa por la cual el Perú, pese su inmenso potencial económico, no puede salir de su situación de país del tercer mundo o, como eufemísticamente lo llaman, país en desarrollo. Por lo tanto, es necesario elaborar un cuadro de estas fricciones sociales y ver cuáles son los conflictos que registra Palma en sus tradiciones y epístolas.

\section{Tiempos violentos en la historia del Perú}

En cuanto a las crisis sociales, estas se observan desde que el hombre comenzó a domeñar la naturaleza; esto sucedió en el período conocido como Arcaico, hace 5000 años. 
Quedan evidencias de fricciones sociales recurrentes en todos los períodos de la historia del Perú, tanto es así que Sempat Asaduorin, al tratar de la historia de los períodos incas e inicios del virreinato, esto es, en los siglos XV y XVI, habla de "guerra permanente" (1983, p. 73). Esta fricción constante la vemos en la formación del imperio incaico; y cuando aún este no se había consolidado. Dice la gente que se escucharon los gritos burlones de las jarjachas que "anunciaban el fin de la generación de los indios"; se avizoraban así tiempos aciagos y, sin más espera, esta racha comenzó con la guerra entre los hermanos Huáscar y Atahualpa, que causó la muerte de Huáscar y de la mayor parte del ejército imperial cuzqueño. El cronista Pedro de Cieza habla de la pérdida de más de cien mil hombres de guerra (1986, p. 227). Y mientras el Perú se desangraba, la hueste hispana clavaba la cruz en el suelo andino y Francisco Pizarro golpeaba la picota con su espada ritual de posesión en el momento que fundaba, cerca al mar y casi a la vera del río Chira, la villa de San Miguel en el valle de Tangarará. Después de este acto, que fue en 1532, la hueste indiana prosiguió hasta Cajamarca, donde tendieron una celada a Atahualpa, lo capturaron y, meses después, lo asesinaron. En este panorama caótico, la hueste hispana marchó al sur entre pequeños focos de rebelión y grandes alianzas como la que los españoles celebraron con los huancas, factor estratégico principal en la caída total del imperio inca. Desde entonces la guerra no se detuvo hasta mediados del siglo XVI, después del triunfo de Pedro La Gasca contra Gonzalo Pizarro, de la llegada del segundo virrey del Perú, don Antonio de Mendoza, en 1551, y de la imposición plena del Virreinato de Nueva Castilla o Perú. Las fricciones que relata Palma en "Los Caballeros de la Capa" corresponden a la última fase de este primer período de fricción social, es decir, desde 1450 hasta 1550.

Otro lapso bélico se produjo desde la década del cuarenta del siglo XVIII con el levantamiento de José Santos Atahualpa y se 
prolonga, aunque con otros protagonistas, hasta la batalla de Quinua y la Capitulación de Ayacucho en 1824. En este lapso de casi un siglo de duración, hubo concurrencias coetáneas o muy cercanas de hechos violentos contra la administración colonial que culminaron con la guerra contra el sistema colonial.

El tercer lapso, aunque breve, es el que se produjo en la segunda mitad del siglo XIX, que estuvo plagado de confrontaciones internas e internacionales. Otro lapso de fricción social en el Perú es el presente, que surge a mediados del siglo XX y se prolonga hasta la actualidad, fricción que tiene varios objetivos y estrategias de confrontación, desde la lucha armada en el "campo y en la ciudad" hasta la violencia verbal y demagógica expresada en los niveles más altos de la política nacional y tiene por fondo el vergonzoso tema de la corrupción.

De los primeros tres lapsos de fricción social (1450-1900), encontramos muchas tradiciones con temas referidos a enfrentamientos sociales; en el último lapso aquí señalado, Palma ya no estaba entre nosotros. En las tradiciones con temas de fricción social, Ricardo Palma demostró un profundo conocimiento de la historia del Perú, tanto del espacio como de sus pobladores con sus buenas y malas artes; entendió a plenitud sus ideologías y extrajo de ellas lo mejor o peor y así logró moldear a sus personajes. En esto, Palma es inigualable y hace buena dupla y va de la mano con Pancho Fierro; ambos son los más grandes exponentes del espíritu de la Lima virreinal. Pancho Fierro concibe su obra fundamentalmente con el paisaje urbano limeño que vio y sintió. Palma nació nueve años después de la Capitulación de Ayacucho, ya no había sistema político virreinal, pero los usos y costumbres de Lima virreinal siguieron igual, por eso Palma pudo ver en vivo y en directo a personajes que seguían viviendo con sus usos y costumbres de data colonial: estos son también los personajes que pintó Pancho Fierro. Palma tenía experiencia directa con personas de 
usanza virreinal y, gracias a sus lecturas, supo de la vida social en tiempos del Virreinato. Estos son factores importantes que determinaron su condición de ser uno de los intelectuales de mayor prestigio en el siglo XIX.

\section{3. "Los caballeros de la Capa. Crónica de una guerra civil"}

Palma, como siempre, usando su vasto conocimiento de la historia de la conquista del Perú con gran ingenio y sobrada picardía, nos presenta, en tres partes, uno de los episodios más funestos de inicios de la vida colonial. Nos referimos a la guerra entre Francisco Pizarro y Diego de Almagro, choque cuyas causas las encontramos en la Capitulación de Toledo firmada por Carlos V y la reina Isabel en 1529. En este documento, Carlos V, en reconocimiento a los esfuerzos y valentía de Francisco Pizarro y sus hombres que permitieron ganar para España inmensos e insospechados territorios, le otorgó el título de Gobernador y Capitán general de las tierras descubiertas y por descubrir (Porras, 1944, p. 2434). Además, le dio muchas facilidades; en cambio, a Diego de Almagro, socio y hombre de la logística de la empresa conquistadora, apenas se le menciona. Esa fue la primera causa de la desavenencia entre Francisco Pizarro y Diego de Almagro. Después, con la fundación de San Miguel, la captura y muerte de Atahualpa, cuando se supo de las inmensas riquezas del mundo andino, creció la ambición entre los miembros de la hueste indiana. Luego del fracasado viaje a Chile y de la creación de una nueva gobernación denominada Nueva Toledo, para compensar a Diego de Almagro, los almagristas reclamaron el Cuzco para la nueva gobernación que había sido creada con el fin de amenguar la fricción entre ambos conquistadores. La posesión del Cuzco fue el factor que originó el incendio de la pradera. Ambos bandos se enfrentaron el 6 de abril de 1538 en un lugar conocido como Las Salinas, 
encuentro que perdieron los almagristas y Diego de Almagro fue condenado a la pena del garrote.

Ricardo Palma divide la tradición "Los caballeros de la Capa" en tres partes: la primera que titula "Quienes eran los caballeros de la capa y el juramento que hicieron"; la segunda que denomina "La atrevida empresa que ejecutaron los caballeros de la Capa", y concluye dicha tradición con "El fin del caudillo de los doce caballeros".

La primera parte la inicia con una reunión clandestina en la Ciudad de los Reyes, en una casa a medio hacer perteneciente al almagrista Pedro de San Millán, un 5 de junio de 1541. Esto sucedía tres años después de la batalla de Las Salinas, confrontación en que fue vencido y condenado a muerte Diego de Almagro. El solar de San Millán es un "testigo" que ayuda a recomponer el casquete urbano de la ciudad capital, donde cada cuadra tenía 10000 metros cuadrados y estaba dividida en cuatro solares; por lo tanto, el solar de Pedro de San Millán tenía 2500 metros cuadrados; esta era la medida estándar para las casas de la Ciudad de los Reyes. El solar tenía la dimensión suficiente para la futura construcción de una casa con viso de fijaldía como correspondía a los conquistadores y fundadores de ciudades. El gran solar que menciona Palma cala con la realidad de entonces; eran espacios a medio hacer, la casa de Pedro de San Millán apenas tenía una gran sala y cinco cuartos y un gran espacio sin construir. La ciudad se fundó con planta simétrica, según lo mandaba la real cédula de 1524 dada por Carlos $\mathrm{V}^{1}$, pero el tráfago de la guerra en que se vivía, causaba que los centros poblados no tuvieran un crecimiento regular; la casa de San Millán, conforme la presenta Palma, es un ejemplo. La Ciudad de los Reyes, que aún no era llamada Lima, estaba conformada por algunas casas a medio hacer como la de San

1 Recopilación de Leyes, 1774, 91R. 
Millán; eran viviendas discontinuas, aún no se configuraba plenamente la cuadrícula. La Plaza de Armas era una pampa, el Palacio de gobierno era una casa, por supuesto más acabada que la de Pedro de San Millán, pero aún no tenía completo el viso de fijaldía. La inestabilidad en que se vivía originaba muchos abandonos de las ciudades dejando las casas a medio hacer, por esos fueron las probanzas que dio en más de una oportunidad el Consejo de Justicia y Regimiento, que así se llamaba la actual Municipalidad.

Otro tema de sumo interés dentro de esta primera parte es la condición de los doce caballeros de la capa: nos referimos a Pedro de San Millán, Cristóbal de Sotelo, García de Alvarado, Francisco de Chávez, Martín de Bilbao, Diego Méndez, Juan Rodríguez Barragán, Gómez Pérez, Diego de Hoces, Martín Castillo, Gerónimo de Almagro y Juan Tello (Lockhart, 1987, pp. 25 -50). Aquí conviene decir que a estos señores, por su condición de conquistadores y fundadores de centros poblados, se les consideraba como Caballeros. Felipe II dio una real cédula que convertía a estos primeros españoles en nobles fijosdalgos con opción a usar el título de Don (De Origen Noble). Entonces en esta tradición, Palma comenta con acierto el aspecto social, y mediante el relato nos permite imaginar hombres con sus "penas y contentamientos", lo mismo que sus viviendas a medio hacer. Hay que añadir otro detalle: James Lockhart en su obra Los de Cajamarca solo menciona a Pedro de San Millán, Cristóbal de Sotelo, Francisco de Chávez, Juan Rodríguez y Martín Carrillo, quienes estuvieron en la toma del tambo real de Cajamarca y en la captura de Atahualpa; los demás llegaron inmediatamente después y estuvieron en la fundación de Trujillo (1535) y en la Ciudad de los Reyes (1535). De igual manera, estuvieron en la brega durante el levantamiento de Manco Inca (1536). También acompañaron a Diego de Almagro en la campaña a Chile, que fue un fracaso. Todo este currículo les daba derechos y privilegios, sobre todo a Diego de Almagro. En medio de 
estos conflictos, se fueron madurando las malas intenciones entre los bandos de Pizarro y Almagro, que los llevó a definir sus derechos por medio de las armas. Esto sucedió en un lugar conocido como Las Salinas, cerca del Cuzco, donde ganaron los pizarristas. Diego de Almagro fue condenado a muerte; a otros como los Caballeros de la Capa se les perdonó la vida, pero sus bienes fueron confiscados y con ello se les condenó a la suma pobreza. Palma demuestra un profundo conocimiento de esta fase temprana del período colonial, que le permite la inspiración y el uso de la metáfora cuando habla del uso recíproco de la capa.

En la segunda parte, titulada "La atrevida empresa que ejecutaron los Caballeros de la Capa", Ricardo Palma, más pegado a la historia, relata toda la estratagema almagrista para terminar con la vida de Francisco Pizarro, que la ejecutaron con éxito el 26 de junio de 1541. Aquel día los almagristas asaltaron la casa de Francisco Pizarro y lo asesinaron. Después de relatar este sangriento hecho con meticulosidad, registra otro hecho peculiar: la aparición del primer colectivo popular en la Ciudad de los Reyes, colectivo que se manifiesta mediante el silencio y el hielo ante la presencia de Diego de Almagro. Los hechos sangrientos del día 26 fueron desaprobados por los españoles residentes en la Ciudad de los Reyes demostrando de muchas maneras el repudio contra Almagro, quien comprendiendo el motivo de esa agria actitud tuvo que alejarse e ir a aposentarse en la ciudad de San Juan de la Frontera, en la provincia de Huamanga.

La Corona, que estaba al corriente de lo que sucedía en el Perú, envió a Cristóbal Vaca de Castro a castigar a los que violaban el orden. Cuando el enviado real llegó a estas tierras, los almagristas estaban en la ciudad de San Juan de la Frontera y en el Cuzco. 
Además, Ricardo Palma puntualiza que la discordia fue "inconando" a los almagristas, tanto fue así que por una desavenencia entre Francisco de Chávez y Almagro, Juan de Rada arrestó a Chávez y lo mandó a degollar. Palma también dice que Juan de Rada, gastado por lo años, murió en Jauja; Palma prosigue señalando el enfrentamiento entre almagristas. Cristóbal Vaca de Castro hizo proposiciones de paz, pero todo fue en vano, la suerte estaba echada y el domingo 16 de septiembre de 1542, en Chupas, cerca de San Juan de la Frontera, se produjo el enfrentamiento en que vencieron las tropas reales y el joven Almagro fue condenado a muerte. Así llegaba a su fin la contradicción entre los conquistadores, cuyo origen aparece con nitidez en la misma Capitulación de Toledo en 1529, tres años antes de que comenzara la conquista del Perú. Pero hay algo más en esta tradición: Palma menciona a Diego Méndez y a Gómez Pérez, almagristas que lograron huir después de la derrota en Chupas y vivieron bajo la protección de Manco Inca, pero, iten cuidado con las traidoras serpientes!, esos españoles en una discusión mataron con alevosía a Manco Inca. Este hecho es otro punto nodal en este rosario de hechos sangrientos que dejaron marca y raíces, las cuales emergen de cuando en cuando en nuestros días.

\section{Bibliografía}

Asadourian, C. S. (1983). El sistema colonial, mercado interno, régimen y espacio económico. Lima: Instituto de Estudios Peruanos.

Cieza de León, P. de. (1986). Crónica del Perú, primera parte. Lima: Fondo Editorial de la PUCP.

Lockhart, J. (1987). Los de Cajamarca, un estudio social y biográfico de los primeros conquistadores del Perú. Lima: Ed. Milla Batre, 
Huertas, V. L. (2009). Las injurias del tiempo, desastres naturales en la historia del Perú. Lima: Fondo Editorial de Universidad Ricardo Palma.

Palma, R. (2014). Tradiciones peruanas. Primera y segunda, tomo I, vol. 1. Lima: Fondo Editorial de la Universidad Ricardo Palma.

Porras Barrenechea, R. (1944). Cedulario del Perú. Siglos XVI, XVII y XVIII, tomo I, 1529-1534. Lima.

Recopilación de leyes de los Reinos de Indias. Tomo II Madrid, 1774.

Recibido el 7 de noviembre de 2019 Aceptado el 11 de noviembre de 2019 